\title{
Genetic overlap between type 2 diabetes and major depressive disorder identified by bioinformatics analysis
}

\author{
Hong-Fang Ji ${ }^{1}$, Qi-Shuai Zhuang ${ }^{1}$ and Liang Shen ${ }^{1}$ \\ ${ }^{1}$ Shandong Provincial Research Center for Bioinformatic Engineering and Technique, School of Life Sciences, Shandong \\ University of Technology, Zibo, P. R. China \\ Correspondence to: Hong-Fang Ji, email: jhf@sdut.edu.cn \\ Liang Shen, email: shen@sdut.edu.cn \\ Keywords: type 2 diabetes; major depressive disorder; single nucleotide polymorphisms; bioinformatics; Gerotarget \\ Received: December 20, $2015 \quad$ Accepted: February 15, $2016 \quad$ Published: March 19, 2016
}

\section{ABSTRACT}

Our study investigated the shared genetic etiology underlying type 2 diabetes (T2D) and major depressive disorder (MDD) by analyzing large-scale genome wide association studies statistics. A total of 496 shared SNPs associated with both T2D and MDD were identified at $p$-value $\leq 1.0 \mathrm{E}-07$. Functional enrichment analysis showed that the enriched pathways pertained to immune responses (FC gamma R-mediated phagocytosis, $T$ cell and B cell receptors signaling), cell signaling (MAPK, Wnt signaling), lipid metabolism, and cancer associated pathways. The findings will have potential implications for future interventional studies of the two diseases.

\section{INTRODUCTION}

Type 2 diabetes (T2D) and depression are prevalent chronic diseases and have been serious public health burdens around the world. The comorbidity between T2D and depression is proven by a series of epidemiological evidence [1-3]. Many studies indicate that the associations between T2D and depression are bidirectional and the presence of T2D increases the risk of depression, and vice versa [4-6].

Despite improved understanding of shared origins of T2D and depression has been gained in recent years [7-10], more effort is needed to identify the shared genetic etiology underlying the two diseases. A wealth of large-scale genome wide association studies (GWAS) about T2D and major depressive disorder (MDD) have been produced in the past few years. These GWAS data provide opportunity to investigate the shared genetic etiology of the two diseases. In this study, we performed a bioinformatics analysis on single nucleotide polymorphisms (SNPs) for T2D and MDD on the basis of GWAS meta-analysis data. The overlapped SNPs for T2D and MDD were identified and functional enrichment analysis was then performed. The findings will benefit future mechanistic and interventional studies for both diseases.

\section{RESULTS}

During the identification of SNPs that are associated with MDD or T2D on the basis of independent DIAbetes Genetics Replication And Meta-analysis (DIAGRAM) or Psychiatric Genomics Consortium meta-GWAS statistics, multiple GWAS genetic association $p$-values ranging from $1.0 \mathrm{E}-10$ to $1.0 \mathrm{E}-06$ were set as cutoff criteria. The results were shown in Table 1. It can be seen that when setting at $p$-value of $1.0 \mathrm{E}-06$ as cutoff criteria, the number of identified SNPs is comparable. With improved $p$-values, the number of identified SNPs associated with T2D is significantly larger than MDD as shown in Table 1. Using $p$-value threshold at 1.0E-10, we identified 2496 and 64 SNPs associated with T2D and MDD, respectively (Table $1)$.

Through overlapping the identified SNPs associated with T2D or MDD, we obtained the number of overlapped SNPs associated with both diseases with different $p$-values as cutoff criteria. As shown in Table 1, when setting the threshold at $p$-value $\leq 1.0 \mathrm{E}-09$ and $\leq 1.0 \mathrm{E}-08$, there were 1 and 10 overlapped SNPs between T2D and MDD, respectively. When setting the threshold at $p$-value $\leq$ 1.0E-07, 496 SNPs were identified to be associated with both diseases, which was significantly larger than random chance (overlapped $p$-value of 5.32E-08 and odds ratio $=$ 1.08).

Among the overlapped 496 SNPs, there were 216 SNPs with annotated genes. The functional enrichment 
Table 1: Number of SNPs associated with T2D or MDD and overlapped SNPs associated with both diseases with different p-values as cutoff criteria

\begin{tabular}{|c|c|c|c|}
\hline \multirow{2}{*}{$P$-value threshold } & \multicolumn{2}{|c|}{ Number of SNPS } & \multirow{2}{*}{$\begin{array}{l}\text { Number of } \\
\text { overlapped SNPs }\end{array}$} \\
\hline & T2D & MDD & \\
\hline $1.0 \mathrm{E}-10$ & 2496 & 64 & 0 \\
\hline $1.0 \mathrm{E}-09$ & 4445 & 505 & 1 \\
\hline $1.0 \mathrm{E}-08$ & 11280 & 3943 & 10 \\
\hline $1.0 \mathrm{E}-07$ & 42271 & 27410 & 496 \\
\hline $1.0 \mathrm{E}-06$ & 127905 & 123041 & 3966 \\
\hline
\end{tabular}

analysis of these genes was performed by using the Database for Annotation, Visualization and Integrated Discovery (DAVID) v6.7 [14] to conduct Kyoto Encyclopedia of Genes and Genomes (KEGG) pathway and GO term enrichment analysis. For each of these KEGG pathways, information regarding pathway size, the total number of genes in the pathway, the Benjaminicorrected $p$-value, and the annotated genes were provided in Table 2. We obtained significant enrichment of the annotated genes in 61 KEGG pathways. Notably, we observed that selected enriched KEGG pathways listed in Table 2 pertain to immune responses ( $\mathrm{Fc}$ gamma R-mediated phagocytosis, $\mathrm{T}$ cell receptor signaling, B cell receptor signaling pathway), cell signaling (MAPK, Wnt signaling), lipid metabolism, as well as several cancer associated pathways.

\section{DISCUSSION}

Based on the available meta-GWAS statistic for T2D and MDD, we performed a bioinformatics analysis to explore the shared genetic etiology underlying the two diseases. When setting the threshold at $p$-value $\leq 1.0 \mathrm{E}$ 07, 496 overlapped SNPs were identified to be associated with both T2D and MDD. Further functional enrichment analysis observed $61 \mathrm{KEGG}$ pathways, including immune responses ( $\mathrm{Fc}$ gamma $\mathrm{R}$-mediated phagocytosis, $\mathrm{T}$ cell and $\mathrm{B}$ cell receptors signaling), cell signaling (MAPK, Wnt signaling), lipid metabolism, as well as several cancer associated pathways.

The findings gained supports from previous studies. First, abnormality in the immune and inflammation systems has been reported to be involved in the pathogenesis of both T2D and MDD [11-15]. Several pathways, including Fc gamma R-mediated phagocytosis, $\mathrm{T}$ cell and $\mathrm{B}$ cell receptors signaling, have been observed (Table 2). Oxidative stress caused by excessive reactive oxygen species (ROS) contribute to the pathogenesis of T2D and MDD. As crucial secondary messengers in signal transduction, ROS may also exert significant effect on inflammatory pathways through MAPK activation $[16,17]$. Wnt signal has also been implicated in the development of both T2D and MDD [18-20] In addition, several cancer associated pathways have been found, which may arise from the epidemiologically observed close relationships between diabetes or depression and many types of cancer [21-23].

There are some limitations in our study. First, the functional enrichment analysis of the shared genes of T2D and MDD is based on the accuracy and completeness of KEGG database. Thus, some genes possessing impacts on both diseases but not annotated in KEGG databases are not included. Second, although we employed two most comprehensive large-scale meta-GWAS statistics for T2D and MDD respectively, other GWAS not considered may potentially affect the results.

In summary, through bioinformatics analysis of two most comprehensive large-scale meta-GWAS statistic of T2D and MDD, we identified the overlapped SNPs and performed functional enrichment pathway of the annotated genes. The findings tentatively support the disease concordance between T2D and MDD indicated by epidemiological studies, and also have potential implications for future therapeutic strategies for the two diseases.

\section{MATERIALS AND METHODS}

The identification of SNPs associated with T2D risk was based on the meta-GWAS statistics from the DIAGRAM consortium study, which was generated from a meta-analysis study covering 34,840 cases and 114,981 controls, overwhelmingly of European descent [24]. These T2D statistics provide more than 2 million SNPs on the basis of the calculation using HapMap project. The identification of SNPs associated with MDD was based on the meta-GWAS statistics from the Psychiatric Genomics Consortium study, the largest and most comprehensively genome-wide analysis of MDD yet conducted [25]. This study investigated more than 1.2 million autosomal and $\mathrm{X}$ chromosome SNPs in 18759 independent and unrelated subjects of recent European ancestry (9240 MDD cases and 9519 controls) in the MDD discovery phase. In the MDD replication phase, this study also evaluated 554 SNPs in independent 6,783 MDD cases and 50,695 controls [25]. 
Table 2: Overlapped pathways identified in T2D and MDD.

\begin{tabular}{|c|c|c|c|c|}
\hline Term & $\begin{array}{l}\text { Total } \\
\text { pathway } \\
\text { size }\end{array}$ & $\begin{array}{l}\text { Number of } \\
\text { genes } \\
\text { modulated } \\
\text { by } \\
\text { overlapped } \\
\text { SNPs } \\
\end{array}$ & $\begin{array}{l}\text { Benjamini- } \\
\text { corrected } \\
p \text {-value }\end{array}$ & Genes \\
\hline ECM-receptor interaction & 2 & 2 & $1.36 \mathrm{E}-05$ & HSPG2, COL4A2 \\
\hline Glycerolipid metabolism & 8 & 3 & $5.46 \mathrm{E}-05$ & PPAP2B, DGKB, LIPC \\
\hline Glycerophospholipid metabolism & 8 & 3 & $5.46 \mathrm{E}-05$ & PPAP2B, DGKB, ACHE \\
\hline Ether lipid metabolism & 2 & 1 & $1.36 \mathrm{E}-05$ & PPAP2B \\
\hline Sphingolipid metabolism & 2 & 1 & $1.36 \mathrm{E}-05$ & PPAP2B \\
\hline Fc gamma R-mediated phagocytosis & 3 & 2 & $2.05 \mathrm{E}-05$ & PPAP2B , DNM3 \\
\hline Cytokine-cytokine receptor interaction & 4 & 4 & $2.73 \mathrm{E}-05$ & LEPR, TGFB2, IL2RA, CCL11 \\
\hline Neuroactive ligand-receptor interaction & 6 & 6 & 4.09E-05 & $\begin{array}{l}\text { LEPR, PTGER3, GABRB1, GRM8, } \\
\text { LPAR2, GRIK1 }\end{array}$ \\
\hline Jak-STAT signaling pathway & 3 & 3 & $2.05 \mathrm{E}-05$ & LEPR, SPRED2, IL2RA \\
\hline Adipocytokine signaling pathway & 2 & 2 & $1.36 \mathrm{E}-05$ & LEPR, PPARGC1A \\
\hline Calcium signaling pathway & 5 & 4 & $3.41 \mathrm{E}-05$ & $\begin{array}{l}\text { PTGER3, SLC8A1, CACNA1C, } \\
\text { ITPR2 }\end{array}$ \\
\hline Cell adhesion molecules & 3 & 2 & $2.05 \mathrm{E}-05$ & NEGR1, CNTN1 \\
\hline Wnt signaling pathway & 7 & 6 & $4.77 \mathrm{E}-05$ & $\begin{array}{l}\text { VANGL1, PRICKLE2, CTBP2, } \\
\text { SMAD3, NFATC3, NFATC2 }\end{array}$ \\
\hline Dorso-ventral axis formation & 2 & 2 & $1.36 \mathrm{E}-05$ & NOTCH2, ETV6 \\
\hline Notch signaling pathway & 2 & 2 & $1.36 \mathrm{E}-05$ & NOTCH2, CTBP2 \\
\hline Endocytosis & 4 & 4 & $2.73 \mathrm{E}-05$ & DNM3, IL2RA, USP8, GIT1 \\
\hline MAPK signaling pathway & 6 & 6 & 4.09E-05 & $\begin{array}{l}\text { TGFB2, MRAS, MAP4K2, } \\
\text { CACNA1C, FGF14, NFATC2 }\end{array}$ \\
\hline Cell cycle & 5 & 3 & $3.41 \mathrm{E}-05$ & TGFB2, E2F3, SMAD3 \\
\hline TGF-beta signaling pathway & 3 & 2 & $2.05 \mathrm{E}-05$ & TGFB2, SMAD3 \\
\hline Pathways in cancer & 11 & 9 & 7.5E-05 & $\begin{array}{l}\text { TGFB2, PPARG, E2F3, CTBP2, } \\
\text { IGF1, FGF14, COL4A2, SMAD3, } \\
\text { DCC }\end{array}$ \\
\hline Colorectal cancer & 4 & 3 & $2.73 \mathrm{E}-05$ & TGFB2, SMAD3, DCC \\
\hline Pancreatic cancer & 5 & 3 & $3.41 \mathrm{E}-05$ & TGFB2, E2F3, SMAD3 \\
\hline Chronic myeloid leukemia & 6 & 4 & $4.09 \mathrm{E}-05$ & TGFB2, E2F3, CTBP2, SMAD3 \\
\hline Hypertrophic cardiomyopathy & 7 & 6 & $4.77 \mathrm{E}-05$ & $\begin{array}{l}\text { TGFB2, SLC8A1, TTN, ACTB, } \\
\text { CACNA1C, IGF1 }\end{array}$ \\
\hline Dilated cardiomyopathy & 7 & 6 & $4.77 \mathrm{E}-05$ & $\begin{array}{l}\text { TGFB2, SLC8A1, TTN, ACTB, } \\
\text { CACNA1C, IGF1 }\end{array}$ \\
\hline O-Glycan biosynthesis & 2 & 2 & $1.36 \mathrm{E}-05$ & GALNT2, C1GALT1 \\
\hline Cardiac muscle contraction & 3 & 2 & $2.05 \mathrm{E}-05$ & SLC8A1, CACNA1C \\
\hline $\begin{array}{lll}\begin{array}{l}\text { Arrhythmogenic } \\
\text { cardiomyopathy }\end{array} & \text { right } & \text { ventricular } \\
\end{array}$ & 5 & 4 & $3.41 \mathrm{E}-05$ & SLC8A1, ACTB, CACNA1C, PKP2 \\
\hline Ubiquitin mediated proteolysis & 2 & 2 & $1.36 \mathrm{E}-05$ & FANCL, UBE2R2 \\
\hline PPAR signaling pathway & 2 & 2 & $1.36 \mathrm{E}-05$ & PPARG, EHHADH \\
\hline Huntington's disease & 5 & 4 & $3.41 \mathrm{E}-05$ & $\begin{array}{l}\text { PPARG, DNAH1, PPARGC1A, } \\
\text { CREB5 }\end{array}$ \\
\hline Valine, leucine and isoleucine biosynthesis & 2 & 2 & $1.36 \mathrm{E}-05$ & LARS2, EHHADH \\
\hline Aminoacyl-tRNA biosynthesis & 2 & 2 & $1.36 \mathrm{E}-05$ & LARS2, CARS \\
\hline Axon guidance & 6 & 6 & 4.09E-05 & $\begin{array}{l}\text { ROBO2, UNC5C, ABLIM1 } \\
\text { NFATC3, DCC, NFATC2 }\end{array}$ \\
\hline Tight junction & 3 & 3 & $2.05 \mathrm{E}-05$ & MRAS, ACTB, SYMPK \\
\hline Regulation of actin cytoskeleton & 4 & 4 & $2.73 \mathrm{E}-05$ & MRAS, ACTB, FGF14, GIT1 \\
\hline Insulin signaling pathway & 2 & 2 & $1.36 \mathrm{E}-05$ & PPARGC1A, GCK \\
\hline
\end{tabular}




\begin{tabular}{|l|l|l|l|l|}
\hline Lysosome & 3 & 3 & $2.05 \mathrm{E}-05$ & MANBA, IGF2R, CTSZ \\
\hline Glioma & 2 & 2 & $1.36 \mathrm{E}-05$ & E2F3, IGF1 \\
\hline Prostate cancer & 3 & 3 & $2.05 \mathrm{E}-05$ & E2F3, CREB5, IGF1 \\
\hline Melanoma & 4 & 3 & $2.73 \mathrm{E}-05$ & E2F3, IGF1, FGF14 \\
\hline Bladder cancer & 2 & 1 & $1.36 \mathrm{E}-05$ & E2F3 \\
\hline Small cell lung cancer & 3 & 2 & $2.05 \mathrm{E}-05$ & E2F3, COL4A2 \\
\hline Non-small cell lung cancer & 2 & 1 & $1.36 \mathrm{E}-05$ & E2F3 \\
\hline Chondroitin sulfate biosynthesis & 2 & 2 & $1.36 \mathrm{E}-05$ & DSE, CSGALNACT1 \\
\hline Focal adhesion & 3 & 3 & $2.05 \mathrm{E}-05$ & ACTB, IGF1, COL4A2 \\
\hline Adherens junction & 4 & 3 & $2.73 \mathrm{E}-05$ & ACTB, PTPRB, SMAD3 \\
\hline Vibrio cholerae infection & 5 & 2 & $3.41 \mathrm{E}-05$ & ACTB, KCNQ1 \\
\hline Phosphatidylinositol signaling system & 6 & 2 & $4.09 \mathrm{E}-05$ & DGKB, ITPR2 \\
\hline Type II diabetes mellitus & 2 & 2 & $1.36 \mathrm{E}-05$ & GCK, CACNA1C \\
\hline Maturity onset diabetes of the young & 3 & 3 & $2.05 \mathrm{E}-05$ & GCK, HNF1A, HNF4A \\
\hline Vascular smooth muscle contraction & 2 & 2 & $1.36 \mathrm{E}-05$ & CACNA1C, ITPR2 \\
\hline Long-term potentiation & 2 & 2 & $1.36 \mathrm{E}-05$ & CACNA1C, ITPR2 \\
\hline GnRH signaling pathway & 2 & 2 & $1.36 \mathrm{E}-05$ & CACNA1C, ITPR2 \\
\hline Alzheimer's disease & 2 & 2 & $1.36 \mathrm{E}-05$ & CACNA1C, ITPR2 \\
\hline Oocyte meiosis & 2 & 2 & $1.36 \mathrm{E}-05$ & ITPR2, IGF1 \\
\hline Long-term depression & 2 & 2 & $1.36 \mathrm{E}-05$ & ITPR2, IGF1 \\
\hline VEGF signaling pathway & 2 & 2 & $1.36 \mathrm{E}-05$ & NFATC3, NFATC2 \\
\hline Natural killer cell mediated cytotoxicity & 2 & 2 & $1.36 \mathrm{E}-05$ & NFATC3, NFATC2 \\
\hline T cell receptor signaling pathway & 2 & 2 & $1.36 \mathrm{E}-05$ & NFATC3, NFATC2 \\
\hline B cell receptor signaling pathway & 2 & 2 & $1.36 \mathrm{E}-05$ & NFATC3, NFATC2 \\
\hline
\end{tabular}

To identify SNPs significantly associated with diseases, multiple cutoff $p$-value criteria were employed. SNPs identified by GWAS were compared to identify overlapped SNPs between T2D and MDD. As we know, for a given complex diseases, such as T2D and MDD, individual genetic variants may interpret only a very small amount of genetic risk. Thus, with the aim to more comprehensively identify SNPs with small effect sizes, a "relaxed" cutoff genetic association $p$-value of 1.0E-07 was employed as a criterion for identifying SNPs that are associated with risk for both T2D and MDD.

The location and mapped genes of each shared SNPs for T2D and MDD were obtained via the SingleNucleotide Polymorphism database (dbSNP) at the National Center for Biotechnology Information (NCBI). Then, functional enrichment analysis of obtained genes was performed by the DAVID v6.7 [26] to perform KEGG pathway and GO term enrichment analysis. The significance of pathway was calculated by statistical method of hypergeometric distribution, and $P$-value of 0.05 was set as the threshold of significance. Significant pathways and GO terms identified in enrichment analysis were compared between T2D and MDD to investigate shared pathways of these two disorders.

\section{ACKNOWLEDGMENTS}

This work was supported in part by the National
Natural Science Foundation of China (Grant No. 31370745), Shandong Provincial Natural Science Foundation for Distinguished Young Scholars (Grant No. JQ201508) and Key program of Shandong Provincial Natural Science Foundation (Grant No. ZR2015JL010).

\section{CONFLICTS OF INTEREST}

The authors declared no potential conflicts of interest.

\section{REFERENCES}

1. Roy T and Lloyd CE. Epidemiology of depression and diabetes: a systematic review. J Affect Disord. 2012; 142:S8-S21.

2. Lloyd CE, Roy T, Nouwen A and Chauhan AM. Epidemiology of depression in diabetes: international and cross-cultural issues. J Affect Disord. 2012; 142:S22-S29.

3. Corriere $\mathrm{M}$, Rooparinesingh $\mathrm{N}$ and Kalyani RR. Epidemiology of diabetes and diabetes complications in the elderly: an emerging public health burden. Curr Diab Rep. 2013; 13:805-813.

4. Nichols GA and Brown JB. Unadjusted and adjusted prevalence of diagnosed depression in type 2 diabetes. Diabetes Care. 2003; 26:744-749

5. Maraldi C, Volpato S, Penninx BW, Yaffe K, Simonsick 
EM, Strotmeyer ES, Cesari M, Kritchevsky SB, Perry S, Ayonayon $\mathrm{HN}$ and Pahor M. Diabetes mellitus, glycemic control, and incident depressive symptoms among 70to 79-year-old persons: the health, aging, and body composition study. Arch Intern Med. 2007; 167:1137-1144.

6. Mezuk B, Eaton WW, Albrecht S and Golden SH. Depression and type 2 diabetes over the lifespan: a metaanalysis. Diabetes Care. 2008; 31:2383-2390.

7. Petrak F, Baumeister H, Skinner TC, Brown A and Holt RI. Depression and diabetes: treatment and health-care delivery. Lancet Diabetes Endocrinol. 2015; 3:472-485.

8. Moulton CD, Pickup JC and Ismail K. The link between depression and diabetes: the search for shared mechanisms. Lancet Diabetes Endocrinol. 2015; 3:461-471.

9. Fisher EB, Chan JC, Nan H, Sartorius N, Oldenburg B. Co-occurrence of diabetes and depression: conceptual considerations for an emerging global health challenge. J Affect Disord. 2012; 142 Suppl:S56-66.

10. Musselman DL, Betan E, Larsen H, Phillips LS. Relationship of depression to diabetes types 1 and 2: epidemiology, biology, and treatment. Biol Psychiatry. 2003; 54:317-329.

11. Kalupahana NS, Moustaid-Moussa N and Claycombe KJ. Immunity as a link between obesity and insulin resistance. Mol Aspects Med. 2012; 33:26-34.

12. Calle MC and Fernandez ML. Inflammation and type 2 diabetes. Diabetes Metab. 2012; 38:183-91.

13. Dantzer R, O'Connor JC, Freund GG, Johnson RW and Kelley KW. From inflammation to sickness and depression: when the immune system subjugates the brain. Nat Rev Neurosci. 2008; 9:46-56.

14. Maes M, Yirmyia R, Noraberg J, Brene S, Hibbeln J, Perini G, Kubera M, Bob P, Lerer B, Maj M. The inflammatory and neurodegenerative (I\&ND) hypothesis of depression: leads for future research and new drug developments in depression. Metab Brain Dis. 2009; 24:27-53.

15. Rawdin BJ1, Mellon SH, Dhabhar FS, Epel ES, Puterman E, Su Y, Burke HM, Reus VI, Rosser R, Hamilton SP, Nelson JC, Wolkowitz OM. Dysregulated relationship of inflammation and oxidative stress in major depression. Brain Behav Immun. 2013; 31:143-152.

16. Evans JL, Goldfine ID, Maddux BA and Grodsky GM. Oxidative stress and stress-activated signaling pathways: a unifying hypothesis of type 2 diabetes. Endocr Rev. 2002; 23:599-622.
17. Bakunina N, Pariante CM and Zunszain PA. Immune mechanisms linked to depression via oxidative stress and neuroprogression. Immunology 2015; 144:365-373.

18. Bordonaro M. Role of Wnt signaling in the development of type 2 diabetes. Vitam Horm. 2009; 80:563-581.

19. Voleti B and Duman RS. The roles of neurotrophic factor and Wnt signaling in depression. Clin Pharmacol Ther. 2012; 91:333-338.

20. Niciu MJ, Ionescu DF, Mathews DC, Richards EM and Zarate CA Jr. Second messenger/signal transduction pathways in major mood disorders: moving from membrane to mechanism of action, part I: major depressive disorder. CNS Spectr. 2013; 18:231-241.

21. Giovannucci E, Harlan DM, Archer MC, Bergenstal RM, Gapstur SM, Habel LA, Pollak M, Regensteiner JG, Yee D. Diabetes and cancer: a consensus report. Diabetes Care. 2010; 33:1674-1685.

22. Larsson SC, Mantzoros CS and Wolk A. Diabetes mellitus and risk of breast cancer: a meta-analysis. Int J Cancer. 2007; 121:856-862.

23. Raison CL and Miller AH. Depression in cancer: new developments regarding diagnosis and treatment. Biol Psychiatry. 2003; 54:283-294.

24. Morris AP, Voight BF, Teslovich TM, Ferreira T, Segrè AV, Steinthorsdottir V, Strawbridge RJ, Khan H, Grallert H, Mahajan A, Prokopenko I, Kang HM, Dina C, Esko T, Fraser RM, Kanoni S, et al. Large-scale association analysis provides insights into the genetic architecture and pathophysiology of type 2 diabetes. Nat Genet. 2012; 44: 981-990.

25. Major Depressive Disorder Working Group of the Psychiatric GWAS Consortium, Ripke S, Wray NR, Lewis CM, Hamilton SP, Weissman MM, Breen G, Byrne EM, Blackwood DH, Boomsma DI, Cichon S, Heath AC, Holsboer F, Lucae S, Madden PA, Martin NG, et al. A mega-analysis of genome-wide association studies for major depressive disorder. Mol Psychiatry. 2013; 18:497511.

26. Huang da W, Sherman BT and Lempicki RA. Systematic and integrative analysis of large gene lists using DAVID bioinformatics resources. Nat Protoc. 2009; 4:44-57. 